\title{
Retraction: The Influence of Cacheable Models on E-Voting Technology
}

\author{
Chen-shin Chien ${ }^{1}$ and Jason Chien ${ }^{2}$ \\ ${ }^{1}$ Department of Industrial Education, \\ National Taiwan Normal University, Taipei County, Taiwan \\ chiendoc@ntnu.edu.tw \\ ${ }^{2}$ China Unversity of Science and Technology Computing Center \\ China Unversity of Science and Technology, Taipei County, Taiwan \\ jason034@cc. cust. edu. tw
}

Several conference proceedings have been infiltrated by fake submissions generated by the SCIgen computer program. Due to the fictional content the chapter "The Influence of Cacheable Models on E-Voting Technology" by "Chen-shin Chien and Jason Chien" has been retracted by the publisher. Measures are being taken to avoid similar breaches in the future. 